\title{
PI 7-I 7. Newborn mice vaccination with rBCG:HIVA + MVA:HIVA enhances HIV-I-specific immune responses. Influence of age and immunization routes
}

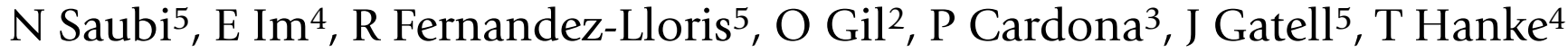 \\ and J Joseph*1,5
}

\begin{abstract}
Address: ${ }^{1}$ MRC Human Immunology Unit, Weatherall Institute of Molecular Medicine, Oxford, UK, ${ }^{2}$ Unitat Tuberculosi Experimental Institut "Germans Trias i Pujol", Badalona, Spain, ${ }^{3}$ Unitat Tuberculosi Experimental, Institut "Germans Trias i Pujol", Badalona, Spain, ${ }^{4}$ MRC Human Immunology Unit, Weatherall Institute of Molecular Medicine, Oxford, UK and ${ }^{5}$ Infectious Diseases Department AIDS Research Unit, Hospital Clinic-IDIBAPS/HIVACAT, Barcelona, Spain

* Corresponding author
\end{abstract}

from AIDS Vaccine 2009

Paris, France. 19-22 October 2009

Published: 22 October 2009

Retrovirology 2009, 6(Suppl 3):P299 doi:I0.I 186/1742-4690-6-S3-P299

This abstract is available from: http://www.retrovirology.com/content/6/S3/P299

(c) 2009 Saubi et al; licensee BioMed Central Ltd.

\section{Background}

It has been demonstrated by several groups that the use of rBCG expressing HIV immunogens is a good priming vector for vaccinia based HIV vaccines, mainly in the prevention of mother-to-child HIV transmission. Our group has shown in Balb/C mice that rBCG:HIVA can both prime novel and boost preexisting HIV-1 specific cellular immune responses. In this study we have evaluated the HIV specific cellular immune responses induced after newborn and adult mice immunization using different routes with rBCG:HIVA prime and MVA:HIVA boost.

\section{Methods}

We have inoculated $10^{6} \mathrm{cfu}$ of rBCG:HIVA to adult mice ( 7 weeks old) by intradermal and subcutaneous route, and 2 $\times 10^{6} \mathrm{cfu}$ to newborn mice (7 days old) by subcutaneous route. $10^{6} \mathrm{pfu}$ of MVA:HIVA was inoculated intramuscularly at 14 weeks post-BCG inoculation, and 3 weeks later the animals were sacrificed. The specific HIV cellular immune responses were analyzed in spleen cells by Intracellular cytokine staining (ICS) and in vitro CTL activity. The body weight of the newborn mice was weekly recorded.

\section{Results}

The frequencies of HIV-specific CD8+ T-cells producing IFN- $\gamma$ was higher in adult mice vaccinated intradermally $(2.18 \%)$ and lower in adult $(1,02 \%)$ and newborn mice $(0,59 \%)$ vaccinated subcutaneously. In all cases the IFN- $\gamma$ production was significantly higher when mice were primed with rBCG:HIVA compared with BCGwt. When the HIV specific CTL activity was assessed, no clear difference was observed between intradermal and subcutaneous route in adult mice, but the frequencies of specific killing were higher in newborn mice than in adults. The prime-boost vaccination regimen which includes rBCG:HIVA and MVA:HIVA was safe when inoculated to newborn mice. No differences in body weight evolution were observed.

\section{Conclusion}

The administration of rBCG:HIVA to newborn mice is safe and immunogenic and increased the HIV-specific responses induced by MVA:HIVA vaccine. It might be a good model for infant HIV and tuberculosis bivalent vaccine. 